\title{
CONTRIBUIÇÃO DAS TIC PARA RECUPERAÇÃO DE DADOS SOBRE PRODUTORES DA AGRICULTURA FAMILIAR
}

\author{
Elaine Parra Affonso ${ }^{1,2}$; Cesar Augusto Pinheiro Vitor ${ }^{3,4}$, Ricardo César Gonçalves Sant ${ }^{\prime}$ Ana ${ }^{1}$; Arthur \\ Simão de Andrade ${ }^{5}$ \\ ${ }^{1}$ Universidade Estadual Paulista (UNESP), PPGCI, Marília, SP. ${ }^{2}$ Faculdade de Tecnologia de Presidente Prudente \\ (FATEC) - Centro Paula Souza, Análise e Desenvolvimento de Sistemas, Presidente Prudente, SP. ${ }^{3}$ Gerenciamento de \\ Redes de Computadores - FADAP/FAP TUPÃ, SP. ${ }^{4}$ Eudécio Luiz Vicente - ETEC, Centro Paula Souza, Adamantina, SP. \\ ${ }^{5}$ Universidade Estadual Paulista (UNESP), Administração, Campus Tupã, SP. e-mail: elaine.affonso@fatec.sp.gov.br
}

\section{RESUMO}

Com a evolução das Tecnologias da Informação e Comunicação (TIC) e estudos do ciclo de vida dos dados, nas fases de coleta, armazenamento, recuperação e descarte dos dados, emerge a visualização de dados como um elemento que pode diminuir a distância entre o produtor e o consumidor, em especial na agricultura familiar. A Ciência da Informação pode contribuir desenvolvendo pesquisas e estudos sobre os aspectos envolvidos no ciclo de vida dos dados, facilitando a popularização no acesso a estes conhecimentos. O objetivo deste artigo é apresentar como as TIC podem contribuir na disponibilização da informação sobre o agricultor familiar para os consumidores, apresentando um recurso para visualização de dados. Utilizou como fonte de recuperação dos dados, as informações presentes no sítio do CoDAF (Competências Digitais para Agricultura Familiar), projeto de extensão da UNESP - Campus de Tupã.

Palavras-chave: Informação e Tecnologia; Recuperação, Visualização, Ciclo de Vida dos Dados, Agricultor Familiar.

\section{CONTRIBUTION OF ICT FOR DATA RECOVERY OF AGRICULTURE PRODUCERS}

\begin{abstract}
With the evolution of Information and Communication Technologies (ICT) and studies of the data life cycle phases of collection, storage, retrieval and disposal of data, emerging data visualization as an element that can bridge the gap between the producer and the consumer, especially in family farmers. Information Science can contribute conducting research and studies on the aspects involved in the data life cycle, facilitating the popularization access to this knowledge. The objective of this paper is to show how ICT can contribute to the provision of information about the family farmers to consumers, presenting a resource for data visualization. Used as a source of data recovery, the information present on the site of CoDAF (Digital Skills for Agriculture Producers) extension project at UNESP - Tupa.
\end{abstract}

Keywords: Information and Technology; Recovery, Visualization, Life Cycle Data, Agriculture Producers. 


\section{INTRODUÇÃO}

Com a crescente utilização das TIC e o processo de visualização de dados é possível tornar mais simples e compreensível o acesso à informação.

De acordo com Sant'Ana (2013) a importância ao acesso a dados vem sendo foco de estudo em diversas áreas do conhecimento e apresenta como novo desafio para as áreas mais diretamente ligadas ao uso das TIC e, neste contexto, destaca-se à Ciência da Informação com um papel preponderante. É necessário propor melhorias em todas as fases do processo, desde a coleta até a visualização dos dados.

Com o avanço das TIC, é possível observar vários recursos para a visualização de dados, por meio de gráficos digitais, animações, multimídia, mapas interativos, com o objetivo de apoiar algum contexto informacional (DIAS; CARVALHO, 2007).

Aos poucos os produtores rurais diminuem as barreiras das comunidades separadas dos grandes centros por estradas precárias e sistemas de comunicação ineficientes e busca o mundo digital, mesmos os agricultores familiares, tradicionalmente considerados como excluídos digitalmente, são atendidos por diferentes programas de inclusão digital (ASSAD; PANCETTI, 2009).

Segundo Assad e Pancetti (2009) a inclusão digital de produtores rurais pode trazer muito benefícios, como acesso a informações sobre a previsão de tempo, preços de insumos, produtos, preços de terras e a própria comunicação.

A informação é considerada com uma condição básica para o desenvolvimento econômico juntamente com o capital, o trabalho e a matéria-prima e o que torna a informação especialmente significativa nos dias atuais é a sua natureza digital (CAPURRO; HJORLAND, 2003).

De acordo com Capurro e Hjorland (2003), a informação deve ser definida em relação à necessidade do público-alvo servido pelos especialistas em informação, não de modo universal ou individualista, mas, de modo coletivo ou particular. A Informação pode responder questões importantes relacionadas às atividades do público alvo.

Dentro deste contexto observa-se a importância da disponibilização da informação provenientes da agricultura familiar para o mercado, cabendo às TIC o papel de tornar a visualização das informações mais interativa e intuitiva, de forma que estas sejam apropriadas de forma mais rápida pelo consumidor.

O objetivo deste artigo é apresentar como as TIC podem contribuir na disponibilização da informação sobre agricultura familiar para os consumidores, apresentando um recurso para recuperação de dados. 


\section{O ACESSO AOS DADOS}

Os avanços das TIC causaram uma fase de fascinação particularmente nas três últimas décadas, quando a disseminação da Internet nos países industrializados deu suporte ao sonho de integração mundial dos povos por meio das redes digitais. A utilização intensa das TIC nas mais diversas situações na sociedade proporciona um cenário cheio de oportunidades para construção de ambientes menos assimétricos nas relações entre seus atores (CASTELLS, 2000).

De acordo com Sant'Ana (2013) para conseguir a redução da assimetria informacional é preciso oferecer condições para o seu entendimento, principalmente por meio da apresentação de suas vantagens e tornando a busca por sua utilização mais atrativa para toda a sociedade.

A Ciência da Informação tem o objetivo de estudar metodologias que tornem possível a disponibilização da informação para que sejam apropriadas pelos usuários de forma mais rápida, desta forma a visualização de dados pode contribuir com técnicas para a construção de estruturas visuais com o propósito de acelerar a apropriação de conhecimento por parte do usuário (DIAS; CARVALHO, 2007).

A visualização de dados é igual a qualquer outro tipo de comunicação, o sucesso é definido pela capacidade do seu público em utiliza-los (FRY, 2008).

Destacando ainda que a Ciência da Informação é destinada as questões científicas e a prática profissional voltada para os problemas da comunicação do conhecimento e de seus registros entre os seres humanos, no contexto social, institucional ou individual do uso e das necessidades de informação, sendo considerado um interesse nas modernas tecnologias informacionais (SARACEVIC, 1991).

Neste artigo, apresenta-se um recurso para recuperação de dados sobre os agricultores familiares, contribuindo para a ampliação da percepção pelos produtores das possíveis vantagens que as TIC podem oferecer na aproximação destes com a sociedade e, por conseguinte com seus potenciais consumidores.

No tópico metodologia são apresentadas as fases do ciclo dos dados e a contextualização deste artigo na fase de recuperação.

\section{AGRICULTURA FAMILIAR E AS TIC}

De acordo com Abramovay (1990) a agricultura familiar é aquela em que a gestão, a propriedade e a maior parte do trabalho utilizam mão de obra da própria família nas atividades econômicas. 
Considera que este setor tem seu papel definido na oferta de alimentos, mãode-obra, matérias-primas e excedentes exportáveis ao setor urbano-industrial, e possui grande importância para o país (FRANÇA, DEL GROSSI; MARQUES, 2009).

Segundo Thornton (2003), a internet é uma importante ferramenta que cria oportunidades para o acesso a informação, mas é necessário pensar no processo de adequação individual dessa tecnologia, pois a adesão das TIC inclui fatores como o acesso e o uso.

Um jeito de consolidar a agricultura familiar é agregar valor aos seus produtos, principalmente no fator desenvolvimento e comercialização, que apontam características como o caráter social da agricultura familiar, a territorialidade do local onde esses produtos são fabricados, o sabor originado de alguma característica artesanal do processo produtivo. Estas oportunidades dependem de inovação dos produtores e de condições para superar as restrições da produção individual (BATALHA et al., 2004).

Acredita-se que as TIC têm papel relevante para o desenvolvimento da agricultura, uma vez que permite que os produtores tenham mais acesso à informação e, com isso evite privações, como o próprio isolamento das comunidades rurais (SILVEIRA; SCHWARTZ, 2011).
De acordo com Capurro e Hjorland (2003) a geração, coleta, organização, interpretação, armazenamento, recuperação, disseminação e transformação da informação deve ser baseada em visões/teorias sobre os problemas, questões e objetivos que a informação deverá satisfazer.

Existem iniciativas que buscam promover condições para o uso de recursos das TIC e melhores acessos e divulgações de informações relacionadas aos agricultores familiares, como por exemplo, o Projeto de Extensão da Universidade Estadual Paulista (UNESP), campus de Tupã, Competências Digitais para a Agricultura Familiar (CODAF, 2014).

O CoDAF (2014) é um projeto de extensão que tem como objetivo fornecer alternativas para diminuir os fatores que prejudicam o acesso à informação por parte dos agricultores familiares, como por exemplo a pouca capacitação e baixa familiaridade com tecnologias, sistemas e informação. Por meio de um portal são apresentadas informações próprias dos produtores familiares, como dados de sua propriedade e características de seus produtos, disponibilizando no sítio uma área especialmente para esta finalidade, podendo divulgar seus produtos, boas práticas e responsabilidade socioambiental aplicadas em suas propriedades (CODAF, 2014). 


\section{METODOLOGIA}

Como procedimento metodológico foi realizado um estudo teórico, utilizando pesquisa bibliográfica e o modelo de ciclo de vida dos dados, especificamente a fase de recuperação dos dados.

A Ciência da Informação, por meio do estudo e identificação dos processos e fatores envolvidos no ciclo de vida dos dados, pode contribuir com este processo de democratização do uso dos dados, estruturando tanto a produção científica sobre o tema como contribuindo para divulgação, viabilizando a mediação no acesso a estes conhecimentos (Sant'Ana, 2013).

De acordo com Sant'Ana (2013), a fase de coleta realiza as atividades vinculadas a definição inicial dos dados, como serão obtidos, filtrados e organizados e identificando a estrutura, formato e os meios de descrição que será utilizado. Na fase de armazenamento são realizadas atividades relacionadas ao processamento, transformação, inserção, modificação, migração, transmissão gerando a persistência de dados em um suporte digital. A recuperação é a fase onde o acesso aos dados se realiza, como por exemplo, a consulta e a visualização.

Uma visualização adequada de dados é um tipo de narrativa, oferecendo uma resposta clara a uma pergunta sem detalhes irrelevantes. Centrando-se sobre a intenção original da pergunta pode-se eliminar tais detalhes porque a questão abre um precedente para o que é e não é necessário (FRY, 2008).

Existem técnicas de visualização voltadas para os diversos tipos de dados, cada uma com características próprias. A escolha da técnica a ser aplicada em cada situação depende do tipo de informação que está sendo tratada, suas tarefas e finalidades. (DIAS; CARVALHO, 2007).

Contextualiza-se a seguir o estudo realizado com base no ciclo de vida dos dados (SANT'ANA, 2013).

A obtenção dos dados foi realizada nas páginas do sítio do Projeto CoDAF (2014), que oferece a localização geográfica dos agricultores familiares. A localização geográfica se baseou em coordenadas (longitude e latitude) coletas através deste sítio.

Para a fase de recuperação dos dados foi utilizada uma plataforma que proporciona uma visualização instantânea. A localização dos dados no mapa é interpretada automaticamente por meio das coordenadas geográficas que foi coletada no sítio do CoDAF (2014).

\section{RESULTADOS}

Observa-se que as TIC podem ser utilizadas como um recurso de apoio à 
agricultura familiar, contribuindo com 0 acesso e a divulgação de informações referentes ao agricultor familiar.

Por meio desta interface de recuperação dos dados é possível tornar as informações pertencentes aos produtores familiares mais acessíveis aos consumidores. O acesso a esta informação poderá ser realizado pelo navegador utilizando um computador desktop/notebook ou dispositivo móvel (smartphone).

O recurso será incorporado ao sítio do projeto CoDAF (2014) como um instrumento para recuperar os dados das páginas do produtor familiar.
Este mecanismo utilizado para visualização dos dados geolocalizados pode ser um importante instrumento de divulgação para os agricultores familiares, pois oferece aos consumidores uma forma facilitadora para encontrar a informação desejada.

O recurso proposto propicia ao consumidor localizar os seguintes tipos de dados: Principais produtos cultivados, nome da propriedade, nome do produtor, as coordenadas para geolocalização (longitude e latitude), e-mail; telefone e um link para a página do Projeto CoDAF (2014) onde está disponível a descrição e as principais informações do produtor. (Figura 1).

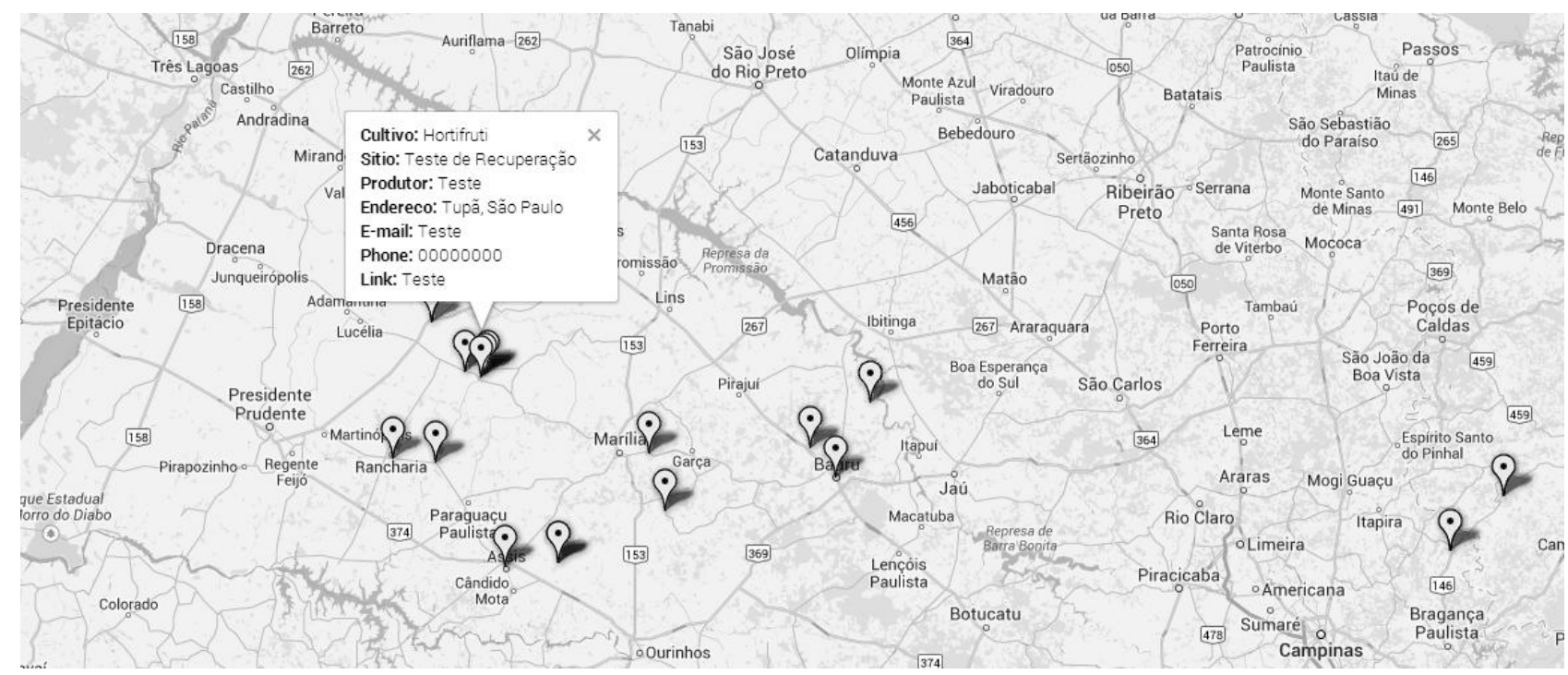

Figura 1. Visualização dos dados do Agricultor Familiar

Ao utilizar o recurso de busca convencional nas páginas do sítio Codaf (2014), que se baseia na digitação de uma palavra-chave, o usuário poderá ter vários documentos para serem analisados e terá que selecionar os resultados desejados, desta forma pode-se utilizar o recurso oferecido para auxiliar na navegação entre os 
resultados, o que diminuirá o tempo de busca deles.

O diferencial ao se utilizar a visualização de dados por mapas é a possibilidade de relacionar vários dados, ao sobrepor o ponteiro do mouse em cada ponto, é possível visualizar os dados de cada produtor, e ter uma visão geral de todos os produtores que tem informações no Codaf (2014).

\section{DISCUSSÕES}

As TIC utilizadas para visualização de dados permitem gerar informações para 0 público interessado, desta forma o recurso apresentado possibilita ao consumidor ter acesso à informação dos pontos de venda e dos tipos de produtos oferecidos (hortaliças, frutas, legumes), visto que muitas vezes a sociedade tem dificuldades de encontrar produtos com qualidade e com um preço atrativo.

O uso de mapas para a visualização de dados pode ser útil quando se deseja apresentar ao consumidor estruturas visuais que melhor representam os dados, realizado a partir da necessidade do consumidor de obter informações sobre um assunto específico.

Observa-se que a tecnologia pode contribuir para diminuir a distância entre o urbano e o rural, possibilitando um maior contato entre produtor e consumidor.

\section{CONCLUSÕES}

Com este trabalho buscou-se estudar alternativas para o uso das TIC para e pelo agricultor familiar oferecendo recursos que possam ser acessados independentes da plataforma tecnológica, possibilitando contribuir com a disponibilização de informações de interesse da sociedade.

As TIC podem contribuir de forma substancial para a Agricultura Familiar, permitindo a recuperação de dados sobre o produtor.

Assim, os resultados deste trabalho reforçam a importância da utilização das TIC na redução da assimetria informacional nas relações em que a agricultura familiar esta inserida, proporcionando uma redução da distância entre o produtor e o consumidor.

\section{REFERÊNCIAS}

ABRAMOVAY, Ricardo. De camponeses a agricultores: Paradigmas do capitalismo em questão. 1990. Tese (Doutorado) - Unicamp SP.

ASSAD, Leonor; PANCETTI, Alessandra. A silenciosa revolução das TIC na agricultura. Com Ciência, n.110., 2009. Disponível em: http://comciencia.scielo.br/scielo.php?script =sci_arttext\&pid=S1519$76542009000600005 \&$ lng=pt\&nrm=iso. Acesso em: ago 2014.

BATALHA, M. O. et al. Tecnologia de gestão e agricultura familiar. CONGRESSO BRASILEIRO DE ECONOMIA E SOCIOLOGIA RURAL, 42., 2004, Cuiabá. Anais... Cuiaba: [s.n.], 2004. 
CAPURRO, Rafael; HJORLAND, Birger. The Concept of Information: theorizing information and information use. Annual Review of informatiocn Science and Technology, v. 37, cap. 8, p. 343-411, 2003.

CASTELLS, Manuel. A Sociedade em rede. São Paulo : Paz e Terra, 2000. v. 1.: A era da informação: economia, sociedade e cultura.

CoDAF. Competências digitais para a agricultura familiar. Disponível em: http://codaf.tupa.unesp.br. Acesso em: ago 2014.

DIAS, M. P.; CARVALHO, J.O.F de. A Visualização da informação e a sua contribuição para a Ciência da Informação. Revista de Ciência da Informação, v. 8 n. 5, out. 2007. Disponível em: http://www.dgz.org.br/out07/Art 02.htm.

Acesso em: ago 2014

FRANÇA, C. G de; DEL GROSSI, M. E,; MARQUES, V. P. M. de A (Org). Censo Agropecuário 2006 e a Agricultura familiar no Brasil. Brasília: MDA, 2009.

FRY, Ben. Visualizing data. U.S.A.: O'Reilly Media, 2008.

SANT'ANA, R. C. G. Ciclo de vida dos dados e o papel da ciência da informação. In: ENCONTRO NACIONAL DE PESQUISA EM CIÊNCIA DA INFORMAÇÃO, 14., 2013, Florianópolis. Anais... Disponível em: http://enancib.sites.ufsc.br/index.php/enanci b2013/XIVenancib/paper/viewFile/284/319.

Acesso em: ago 2014

SARACEVIC, Tefko. Information science: historical, empirical and theoretical perpectives. In: INTERNATIONAL CONFERENCE ON CONCEPTIONS OF LIBRARY, 1., 1991, Tampere. Electronic Proceedings... Tampere: University of Tampere, 1991.

SILVEIRA, A. C. M; SCHWARTZ, Clarissa. TIC e relações afetivo-produtivas na agricultura familiar: enfrentando $O$ isolamento e a exclusão digital. In: CIRCUITO DE DEBATES ACADÊMICOS, 1., 2011. Anais... [S.I.]: [s.n.], 2011.

THORNTON, R. El agricultor, Internet y las barreras a su adopción. In: THORNTON, R.; CIMADEVILLA, G. (Ed.). La extensión rural en debate: concepciones, retrospectivas, cambios y estratégias para el Mercosur. Buenos Aires: Inta, 2003. p. 323-344.

Recebido para publicação em 19/08/2014 Revisado em 23/08/2014

Aceito em 25/08/2014 